\title{
Rise and fall of plant mitochondria
}

\section{Peter Nick}

Published online: 1 April 2010

(C) Springer-Verlag 2010

The acquisition of mitochondria by an endosymbiotic event represented a breakthrough on the way towards modern eukaryotes and was followed by a thorough "domestication" of the endosymbiont by extensive gene transfer and control of endosymbiotic division by the host cell. This domestication has been much more profound as compared with plastidic endosymbionts. For instance, whereas plastid division is still dependent on a prokaryotic (although nuclear encoded) tubulin homologue, FtsZ, mitochondrial division has been much more tightly linked with the division machinery of the host cell. But, mitochondria have not only been placed under almost complete control by the host cell with respect to their genesis, they have been intimately integrated into hostcell signaling. Mitochondria are key players in the regulation of programmed cell death all over animals, fungi, and plants. When the mitochondrial transmembrane potential is impaired due to environmental stresses, they will produce reactive oxygen species, that, if not scavenged, cause not only cell damage, but are important components of the signaling culminating in apoptotic cell death (Balaban et al. 2005). Several contributions of the current issue highlight the generation of plant mitochondria as well as their decay in the context of programmed cell death.

The work by Imoto et al. in the current issue investigates mitochondrial division in the model organism Cyanidioschyzon merolae, a primitive red alga, which contains only a single mitochondrion and a single plastid per cell, whose

\section{P. Nick $(\square)$}

Botanisches Institut,

Lehrstuhl 1 Molekulare Zellbiologie, Kaiserstrasse 2,

76131 Karlsruhe, Germany

e-mail: peter.nick@bio.uka.de division can be highly synchronized by a light/dark cycle. This allows the analysis of the organelle division in the context of the host cell, which is difficult in other models, where organelles divide randomly. The authors examined the roles of microtubules in these cycles by the use of a microtubule-eliminating drug, oryzalin. They can show that the divisions of the nucleus and the mitochondrion were dependent on microtubules, whereas that of the plastid was not. They provide evidence that not only the nuclear, but also the mitochondrial division, is organized by the spindlepole body, illustrating by direct comparison the progressive loss of autonomy between the two organelles and the replacement of the ancestral division machinery by that of the host cell.

The fate of mitochondria during signal-dependent aging is investigated by Kirasak et al. (in this issue) using petal maturation in the orchid Dendrobium cv. Lucky Duan. Floral maturation is integrated into a complex developmental program, where the ordered decay or reprogramming of the maternal tissue has to be synchronized with embryo development. Following petal cytology with electron microscopy, the authors show that the degradation of mitochondria takes place within the organelle itself and that the degraded mitochondria are not transferred into a lytic vacuole, but merge into a vacuolar structure by themselves. This means that the degradation of mitochondria is organelle-autonomous representing a very advanced stage in the coevolution of host-cell and endosymbiont.

Mitochondrial degradation in the context of programmed cell death is not only interesting with respect to intracellular integration, but is of eminent importance for application. This is illustrated by two publications dealing with cytoplasmic male sterility, an important tool for plant breeding, because it allows the generation of hybrids without cumbersome emasculation in important crops such 
as rice or the Brassicaceae. The work by Shi et al. (in this issue) follows pollen degeneration in a male sterile radish line and demonstrates a series of ultrastructural changes including vacuolation, mitochondrial disintegration, and eventually nuclear decay. The work by $\mathrm{Hu}$ et al. (in this issue) describes two propulations of mitochondria in cytoplasmic male sterility of rice using flow cytometry after staining with rhodamine-123 as monitor for the integrity of mitochondrial transmembrane potential. They can show that hydrogen peroxide can stimulate the formation of mito- chondria, with impaired potential along with a reduction in ATP-synthesis and a decay of mitochondrial DNA. Mitochondria are thus central targets for a biotechnological manipulation of male sterility.

\section{Reference}

Balaban RS, Nemoto S, Finkel T (2005) Mitochondria, oxidants, and aging. Cell 120:483-495 